\title{
Anomalous Tensile Detwinning in Twinned Metallic Nanowires
}

\author{
Guangming Cheng ${ }^{1}$ Yin Sheng ${ }^{2}$, Huajian $\mathrm{Gao}^{2}$ and Yong Zhu ${ }^{1}$ \\ 1. Department of Mechanical and Aerospace Engineering, North Carolina State University, Raleigh, NC \\ 27695, USA \\ 2. School of Engineering, Brown University, Providence, RI 02912, USA
}

Nanowires (NWs) are among the most important building blocks for many applications ranging from nanosensors to energy harvesting/storage to flexible/stretchable electronics, to name a few. Operation and reliability of the NW-based devices call for a thorough understanding of their mechanical behaviors. Dislocation nucleation from free surfaces has been identified as a dominant deformation mechanism in NWs, in contrast to the forest dislocation dynamics in bulk materials. Extensive studies have been reported for defect-free, single crystalline metallic NWs where surface dislocation nucleation is dominant [1-3]. Such NWs exhibit ultrahigh yield strength, but typically with limited or no strain hardening and low tensile ductility due to the absence of effective obstacles within the NWs that could block the movement of dislocations. On the other hand, as-synthesized NWs typically possess different types of preexisting microstructures such as twin boundaries (TBs) [2-4], and there is still a general lack of understanding on how preexisting microstructures interact with the surface-nucleated dislocations and affect the mechanical behaviors of NWs.

Here, based on a recently developed testing platform combining state-of-the-art microelectromechanical system (MEMS) technology and in situ TEM tensile tests, we conduct a systematic investigation of the deformation mechanisms in bi-twinned Ag NWs, each having a single TB running parallel to the NW length direction [5]. We performed in situ TEM tensile testing of individual NWs using a MEMS-based tensile testing stage that allows accurate measurements of both load and displacement simultaneously, as well as real-time imaging of microstructure evolution during deformation [2,4]. Figure 1 shows stressstrain responses and snapshots of microstructure evolution of two typical bi-twinned Ag NWs under tensile tests. Insets in Figs. 1a,b show cross-sectional TEM images of the tested NWs, taken from the undeformed parts (beyond the clamps) after the tensile tests. The two bi-twinned NWs possess different volume ratios, defined as $\mathrm{V}_{\text {small }} / \mathrm{V}_{\text {large, }}$, between the two twin variants (the insets in Figs. 1a,b), 0.56 and 0.19 , respectively.

We identified two fundamental deformation mechanisms in bi-twinned NWs, localized dislocation slip and delocalized tensile detwinning. Localized dislocation nucleation and propagation across the TB led to limited plasticity (3.5\%, Figs. 1a,c) in bi-twinned NWs with balanced volume ratios, while delocalized tensile detwinning deformation resulted in large plasticity with an elongation of 34.5\% (Figs. 1b,d)) in those with small volume ratios, characterized by interaction of multiple dislocations with the original TB. The detwinning process was unexpected in view of no apparent resolved shear stress on the preexisting $\mathrm{TB}$, which was attributed to the novel tensile detwinning mechanism - nucleation of a single crystalline embryo as a result of multiple dislocation interactions on the preexisting TB (step-one detwinning), followed by migration of the unique TB-GB-TB structure (step-two detwinning), as corroborated by MD simulations. Our experimental and theoretical results indicated that the deformation mode of a bi-twinned NW is governed by the step-one detwinning. An energy-based criterion for the anomalous tensile detwinning was proposed, which was able to capture the effects of the volume ratio and cross-sectional aspect ratio on the transition of deformation mechanisms in bi-twinned NWs [6]. 


\section{References:}

[1] J. R. Greer and J. T. M. De Hosson, Progress in Materials Science 56 (2011), 654.

[2] Q. Qin et al, Nature communications 6 (2015), 5983.

[3] S. Narayanan et al, Nano letters 15 (2015), 4037.

[4] T.-H. Chang et al, Extreme Mechanics Letters, 8 (2016), 177.

[5] G. Cheng et al, Physical Review Letters 119 (2017), 256101.

[6] The authors acknowledge funding from the National Science Foundation (NSF) under Award Nos. DMR-1410475 and DMR-1709318, and the use of the Analytical Instrumentation Facility (AIF) at North Carolina State University (NSF Award No. ECCS-1542015).

(a)

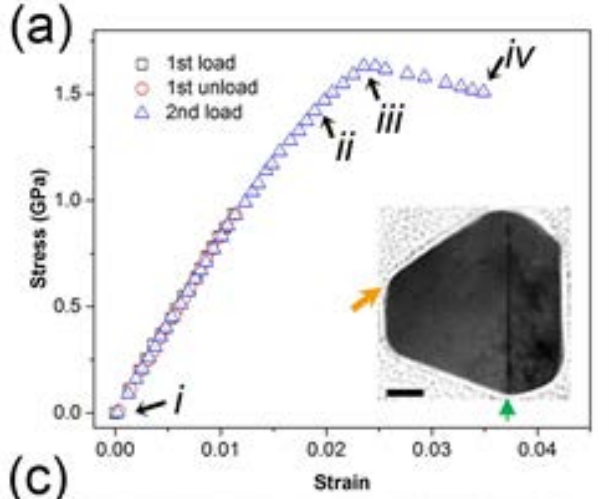

(c)

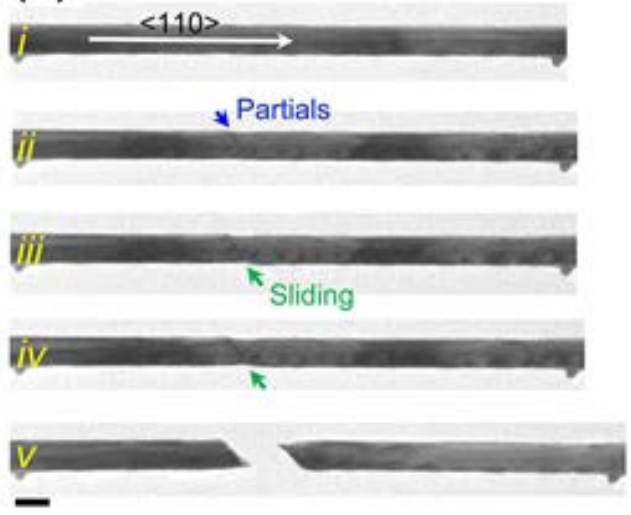

(b)

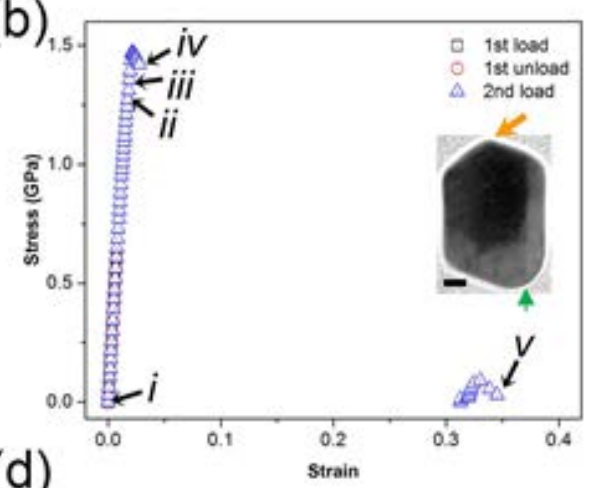

(d)

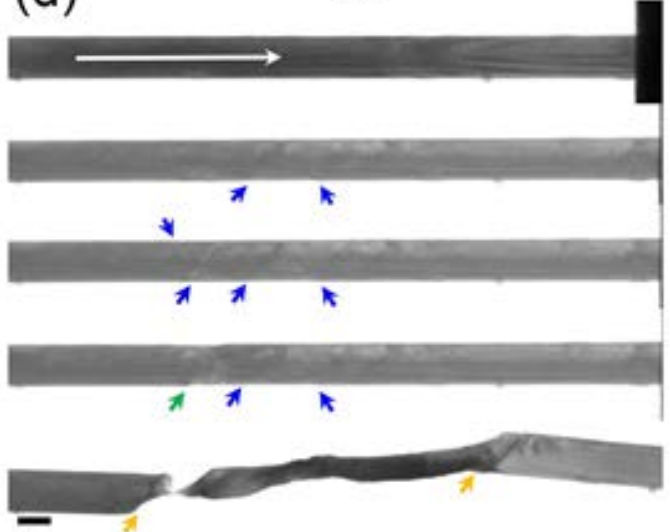

Figure 1. (a,b) Engineering stress-strain curves of bi-twinned Ag NWs with balanced and small volume ratio, respectively. Insets in $(a, b)$ are the corresponding cross-sectional images of the tested NWs (each sectioned from the undeformed part after the test). Location of the TB is marked by a green arrow. Scale bar, $20 \mathrm{~nm}$. (c,d) Snapshots of microstructure evolutions corresponding to (a,b), respectively. The five snapshots in each case correspond to the data points marked in (a,b). Partial dislocations are marked by blue arrows and planar sliding by green arrows. The viewing directions are from the $\langle 1 \overline{1} 0\rangle$ zone axis of the large twin variant in (c,d), which are marked by the orange arrows in the insets in (a,b). The new inclined TBs in (d) are marked by orange arrows. Scale bar, $100 \mathrm{~nm}$. 